\title{
Use of blood biomarkers to screen for obstructive sleep apnea
}

This article was published in the following Dove Press journal:

Nature and Science of Sleep

Wesley Elon Fleming,' JonErik C Holty, ${ }^{2}$ Richard K Bogan, ${ }^{3}$ Dennis Hwang, ${ }^{4}$ Aliya S Ferouz-Colborn, ${ }^{4}$ Rohit Budhiraja, ${ }^{5}$ Susan Redline, ${ }^{5}$ Edith MensahOsman, ${ }^{6}$ Nadir Ishag Osman, ${ }^{6}$ Qing Li, ${ }^{7}$ Armaghan Azad,' Susann Podolak,' Michael K Samoszuk, ${ }^{8}$ Amabelle B Cruz, ${ }^{8}$ Yang Bai, ${ }^{8}$ Jiuliu Lu, ${ }^{8}$ John $S$ Riley, ${ }^{8}$ Paula C Southwick ${ }^{8}$

'Sleep Center Orange County, Irvine, CA, USA; ${ }^{2}$ Stanford Medical School, VA Palo Alto Health Care System, Pulmonary, Critical Care and Sleep Medicine Section, Palo Alto, CA, USA; '3SleepMed Inc., Bogan Sleep Consultants, LLC, Columbia, SC, USA; ${ }^{4}$ Sleep Medicine Department, Southern California Permanente Medical Group, Kaiser Permanente, Fontana Medical Center, Fontana, CA, USA; ${ }^{5}$ Division of Sleep Medicine, Harvard Medical School, Brigham and Women's Hospital, Boston, MA, USA; ${ }^{6}$ EENA Comprehensive Neurology and Sleep Center, Boynton Beach, FL, USA; ${ }^{7}$ South Bend Medical Foundation, New Technology and Test Development, South Bend, IN, USA; ${ }^{8} \mathrm{Clinical}$ Research Department, Beckman Coulter, Inc., Brea, CA, USA
Correspondence: Wesley Elon Fleming Sleep Center Orange County, 4980 Barranca Pkwy, Ste I70, Irvine, CA 92604, USA

Tel +l 9496795510

Fax +19496791080

Email flemingwesley@gmail.com
Purpose: Obstructive sleep apnea (OSA) is a highly prevalent disorder associated with increased risk for cardiovascular disease, diabetes, and other chronic conditions. Unfortunately, up to $90 \%$ of individuals with OSA remain without a diagnosis or therapy. We assess the relationship between OSA and blood biomarkers, and test the hypothesis that combinations of markers provide a characteristic OSA signature with diagnostic screening value. This validation study was conducted in an independent cohort in order to replicate findings from a prior feasibility study.

Patients and methods: This multicenter prospective study consecutively enrolled adult male subjects with clinically suspected OSA. All subjects underwent overnight sleep studies. An asymptomatic control group was also obtained. Five biomarkers were tested: glycated hemoglobin (HbA1c), C-reactive protein (CRP), uric acid, erythropoietin (EPO), and interleukin-6 (IL-6). Results: The study enrolled 264 subjects. The combination of HbA1c+CRP+EPO (area under the curve 0.78) was superior to the Epworth Sleepiness Scale (ESS; 0.53) and STOP-Bang (0.70) questionnaires. In non-obese subjects, the combination of biomarkers $(0.75)$ was superior to body mass index (BMI; 0.61). Sensitivity and specificity results, respectively, were: HbAlc+CRP+EPO (81\% and 60\%), ESS (78\% and 19\%), STOP-Bang (75\% and 52\%), BMI (81\% and 56\%), and BMI in non-obese patients ( $81 \%$ and $38 \%)$.

Conclusion: We verify our hypothesis and replicate our prior feasibility findings that OSA is associated with a characteristic signature cluster of biomarker changes in men. Concurrent elevations of HbA1c, CRP, and EPO levels should generate a high suspicion of OSA and may have utility as an OSA screening tool. Biomarker combinations correlate with OSA severity and, therefore, may assist sleep centers in identifying and triaging higher risk patients for sleep study diagnosis and treatment.

Keywords: obstructive sleep apnea, OSA, screening, diagnosis, biomarkers, CRP, HbA1c, erythropoietin, EPO, uric acid, IL-6

\section{Introduction}

Obstructive sleep apnea (OSA) is a disorder with high prevalence - estimated to occur in $34 \%$ of men and $17 \%$ of women, ${ }^{1}$ afflicting more than 100 million adults worldwide..$^{2,3}$ Unfortunately, less than $30 \%$ of patients currently evaluated by a primary care provider are screened for OSA, with the result that up to $90 \%$ of individuals with OSA remain without a diagnosis or therapy. ${ }^{2-5}$ Patients with untreated OSA are at increased risk for hypertension, cardiovascular disease, heart failure, stroke, obesity, metabolic dysregulation, diabetes mellitus, daytime sleepiness, depression, accidents, and allcause mortality, ${ }^{6-11}$ and are a significant burden on the healthcare system. ${ }^{12,13}$ After treatment, decreases in the risk of chronic health conditions ${ }^{12,14}$ as well as healthcare 
utilization and cost have been reported. ${ }^{15}$ The association between OSA and adverse health consequences has led the American Heart Association and others to suggest that OSA screening be integrated into routine clinical care. ${ }^{6,16}$

Current tools for OSA screening rely on questionnaires with low diagnostic accuracy from low-quality studies, as reported in meta-analyses. ${ }^{17}$ OSA screening measures that are frequently used include the Epworth Sleepiness Scale (ESS) and STOP-Bang questionnaires. The ESS assesses subjective daytime sleepiness but is nonspecific for OSA, was not designed nor validated for OSA screening (yet, remains the most frequently used test, particularly by primary care and other non-sleep-medicine clinicians), is prone to bias, and demonstrates a high false-negative rate. ${ }^{12}$ The STOP-Bang questionnaire, a screening tool specific for OSA, has a relatively high sensitivity but low specificity. ${ }^{12}$ The recent American Academy of Sleep Medicine (AASM) clinical practice guidelines ${ }^{12}$ report that more accurate and user-friendly screening tools, such as blood biomarkers, are needed to better predict OSA diagnosis and severity. A recent review of potential OSA biomarkers ${ }^{18}$ concludes that an optimal screening test should be clinically sensitive, specific, simple, timely, inexpensive, and correlate to disease severity. Furthermore, biomarkers should make pathophysiological sense, reflecting functional changes that accompany OSA. ${ }^{19,20}$ A blood test could potentially meet these criteria, providing risk stratification to prioritize diagnostic sleep study testing and treatment.

Numerous individual OSA blood biomarkers have been studied previously. ${ }^{18,20,21}$ Dysfunctions in metabolic and endocrine systems induced by OSA, chronic inflammation, hypoxemia, sleep fragmentation, and stress are associated with alterations in biomarkers. These biomarkers include glycated hemoglobin (HbA1c), C-reactive protein (CRP), erythropoietin (EPO), interleukin-6 (IL-6), and uric acid. ${ }^{18,21-26}$ Unfortunately, the diagnostic utility of individual biomarkers or combinations of markers is inconclusive in identifying OSA. ${ }^{18,21}$

The objective of this study was to assess the relationship between OSA and five blood biomarkers in patients with a clinical suspicion of OSA, and validate findings from a feasibility study in which 11 biomarkers were assessed. ${ }^{27}$ Both studies (feasibility and validation) were conducted in independent cohorts. Additionally, a hypothesis was tested that combinations of markers are more predictive of OSA than individual markers. Such clusters of OSA-associated biomarkers could provide a characteristic physiologic signature of OSA that may be valuable in OSA screening.

\section{Material and methods}

\section{Study participants}

A multicenter prospective study was conducted to assess the relationship between OSA and endocrine, inflammatory, and metabolic biomarkers in consecutively enrolled adult male patients with a clinical suspicion of OSA; subjects were followed from a general medical clinic population (primary care clinicians $82 \%$ ) to a sleep clinic. Subjects excluded were those with previously diagnosed or treated OSA or currently using steroids, chronic anti-inflammatory drugs, or opioid pain medications. All subjects underwent attended polysomnography in an accredited sleep center, or unattended home sleep apnea testing, as per routine clinical practice. An uncomplicated clinical population was also enrolled as a healthy control group. The study was approved by the institutional review boards or ethics committees at participating centers, and carried out in accordance with The Code of Ethics of the World Medical Association (Declaration of Helsinki). The full title of each ethical review board, and its affiliated institution, is provided in a supplementary Table S1. Written informed consent was obtained from all subjects.

\section{Study sites}

This study was conducted at six institutions: Stanford Medical School/VA Palo Alto Health Care System; Brigham and Women's Hospital/Harvard Medical School (Boston, MA); Southern California Permanente Medical Group/Kaiser Permanente Sleep Center (Fontana, CA, USA); Sleep Center Orange County (Irvine, CA, USA); SleepMed (Columbia, SC, USA); EENA Comprehensive Neurology and Sleep Center (Boynton Beach, FL, USA). Additionally, an uncomplicated asymptomatic clinical population was also recruited from a contract research organization.

\section{Clinical assessment and questionnaires}

Clinical evaluation included a sleep history, physical examination, and follow up under the supervision of a board-certified sleep medicine specialist. Questionnaires were completed and medical histories were obtained before the subjects underwent sleep studies. Daytime sleepiness was assessed by the Epworth Sleepiness Scale (ESS); ESS scores range from 0 to 24 ; a score $>10$ indicates excessive daytime sleepiness. The STOP-Bang questionnaire was also administered; this OSA screening tool consists of 4 questions relating to symptoms (snoring, tired, observed, blood pressure) and 4 questions relating to physical attributes (BMI, age, neck circumference, gender), with scores ranging from 0 to 8 . A score $\geq 3$ suggests increased risk of OSA. 


\section{Diagnostic sleep studies}

Participants underwent attended in-laboratory polysomnography (PSG) in an accredited sleep center, or unattended Type 3 home sleep apnea testing (HSAT) with a technically adequate device approved by the AASM under the supervision of a board-certified sleep medicine physician, who followed published recommendations, methods and scoring guidelines. ${ }^{12,28}$ Patients with comorbidities or symptoms of other significant sleep disorders underwent PSG, whereas patients without complications underwent HSAT. The sleep recordings were manually scored by a registered polysomnographic technologist and subsequently reviewed by a board-certified sleep physician. The apnea-hypopnea index (AHI) was used in the diagnosis and assessment of severity. Respiratory events (apneas and hypopneas) were scored per the AASM Scoring Manual v2.3. ${ }^{28}$ Severity thresholds for mild, moderate, and severe OSA were AHI $\geq 5$, AHI $\geq 15$, and $\mathrm{AHI} \geq 30$, respectively. Other parameters measured were minimum and average oxygen saturation as well as percentage of time with oxygen saturation $<90 \%$.

\section{Sample collection and biomarker testing} Five biomarkers were tested: $\mathrm{HbAlc}$, high sensitivity CRP, IL-6, uric acid, and EPO. These were selected as the most predictive markers from a feasibility study in which 11 biomarkers were assessed. ${ }^{27}$ Clinicians interpreting the diagnostic sleep studies were blinded to the biomarker results. Blood samples were collected in EDTA and serum (SST) tubes at the time of study enrollment prior to initiation of therapy, within 1 day (median) of the sleep study (interquartile range [IQR] 0-3 days). Whole blood samples were stored at $-20^{\circ} \mathrm{C}$ or colder, and shipped to a central laboratory for $\mathrm{HbA} 1 \mathrm{c}$ testing. Serum for all other testing was separated from cells, dispensed into cryo-tubes, stored at $-20^{\circ} \mathrm{C}$ or colder, and shipped to a central laboratory for testing. Samples were tested on the following systems: Beckman Coulter AU System (HbAlc, CRP, and uric acid), and Beckman Coulter UniCel ${ }^{\circledR}$ Access $^{\circledR} 2$ System (EPO and IL-6).

\section{Statistical methods}

Descriptive statistics for each variable were two-tailed Student's $t$-tests or Mann-Whitney tests for continuous variables, and chi-square tests for dichotomous variables. Spearman correlation coefficients were used. To assess clinical performance for OSA detection, cutoffs were evaluated using receiver operating characteristic (ROC) curves. Sensitivity and specificity as well as area under the curve (AUC) and 95\% CI were determined. Statistical analyses were conducted using SAS System software (version 9.3, SAS Institute Inc., Cary, NC, USA) and Analyse-it (version 2.26, Analyse-it Software, Ltd., Leeds, United Kingdom).

\section{Biomarker combinations}

The algorithm developed investigated the discriminative power of five biomarkers in the following manner: two- and three-biomarker combinations were examined with a linear classification model with two optimization methods (linear model - linear value; linear model - log value). Either the original values or the $\log$ values of the biomarkers were treated as independent variables of a predictive model and the output of the model was an algorithm-derived numerical score indicating the severity of OSA.

\section{Results Study population characteristics}

Two-hundred and sixty-four male subjects were enrolled in this study. Patients with AHI $\geq 5$ were considered to have OSA ( $\mathrm{n}=221$ ), and patients with AHI $<5$ were enrolled as the Non-OSA control group ( $\mathrm{n}=31$; median age 38 ; median BMI 29). Furthermore, an uncomplicated asymptomatic clinical population was enrolled as a healthy control group $(n=12$; median age 37 years; median BMI 24) in whom occult OSA was excluded, in order to replicate findings from the feasibility trial ( $\mathrm{n}=20$ healthy controls) that correlated biomarkers with severity of disease. Study population characteristics stratified by diagnosis are shown in Table 1 . In this study population, $48 \%$ reported daytime sleepiness (ESS $>10$ ), $97 \%$ had a STOP-Bang score $\geq 3$, and $72 \%$ had a STOP-Bang score $\geq 5$.

\section{Biomarkers and measures of OSA severity: correlations}

The most significant associations of biomarkers with OSA severity indices were for CRP and HbAlc. Specifically, statistically significant correlations were observed for CRP vs AHI $(r=0.40, p<0.0001)$, CRP vs minimum oxygen saturation $(r=-0.36, p<0.0001)$ and CRP vs sleep time with oxygen saturation $\leq 90 \%(r=0.41, p<0.0001)$. Significant results were also observed for HbA1c vs AHI $(r=0.29$, $p<0.0001), \mathrm{HbA} 1 \mathrm{c}$ vs minimum oxygen saturation $(r=-0.31$, $p<0.0001)$, and $\mathrm{HbA} 1 \mathrm{c}$ vs sleep time with oxygen saturation $\leq 90 \%(r=0.28, p<0.0001)$. Moreover, statistically significant correlations were observed for EPO vs AHI ( $r=0.17$, $p=0.008)$, EPO vs minimum oxygen saturation ( $r=-0.17$, $p=0.008$ ), and EPO vs sleep time with oxygen saturation $\leq 90 \%(r=0.16, p=0.02)$. 
Table I Study population characteristics by diagnosis

\begin{tabular}{|c|c|c|c|c|c|}
\hline Parameter & Characteristics & $\begin{array}{l}\text { Total } \\
(n=264)\end{array}$ & $\begin{array}{l}\text { Non-OSA } \\
(n=43)\end{array}$ & $\begin{array}{l}\text { OSA } \\
(n=221)\end{array}$ & $p$-value \\
\hline Age & Age (years) & $45.0(36.0-53.0)$ & $38.0(34.0-47.0)$ & $47.0(37.0-54.0)$ & $<0.05$ \\
\hline \multirow[t]{4}{*}{ Race } & White & $168 / 264(63.6 \%)$ & 31 & 137 & 0.883 \\
\hline & Black & $40 / 264(15.2 \%)$ & 6 & 34 & \\
\hline & Asian/Pacific Islander & $29 / 264(11.0 \%)$ & 4 & 25 & \\
\hline & Other/unknown & $27 / 264(10.2 \%)$ & 7 & 20 & \\
\hline \multirow[t]{3}{*}{ Baseline characteristics } & BMI & $30.9(28.0-36.1)$ & $27.1(24.3-29.8)$ & $31.9(28.7-37.1)$ & $<0.05$ \\
\hline & Neck circumference (inches) & $17.0(16.0-18.0)$ & $16.0(15.0-17.0)$ & $17.0(16.0-18.3)$ & $<0.05$ \\
\hline & ESS & $10(6-15)$ & II (7-I5) & $10(6-15)$ & 0.601 \\
\hline \multirow[t]{3}{*}{ Comorbidities } & Obesity (BMI $\geq 30)$ & |49/25| (59.4\%) & $\mid \mathrm{I} / 3 \mathrm{|}(35.5 \%)$ & $138 / 220(62.7 \%)$ & $<0.05$ \\
\hline & Hypertension & $95 / 251$ (37.9\%) & II/3I (35.5\%) & $84 / 220(38.2 \%)$ & 0.529 \\
\hline & Diabetes mellitus & $30 / 251$ (12.0\%) & $\mathrm{I} / 3 \mathrm{I}(3.2 \%)$ & $29 / 220$ (13.2\%) & 0.218 \\
\hline
\end{tabular}

Notes: Data are expressed as median (interquartile range), or as $\mathrm{n} / \mathrm{N}(\%)$. $p$-values are shown for each characteristic. The $p$-value for race represents the relationship between diagnosis and all levels of the characteristic. Hypertension is defined as sustained elevation of blood pressure exceeding $140 / 90 \mathrm{mmHg}$, or high blood pressure requiring medication.

Abbreviations: BMI, body mass index; ESS, Epworth Sleepiness Scale; OSA, obstructive sleep apnea.

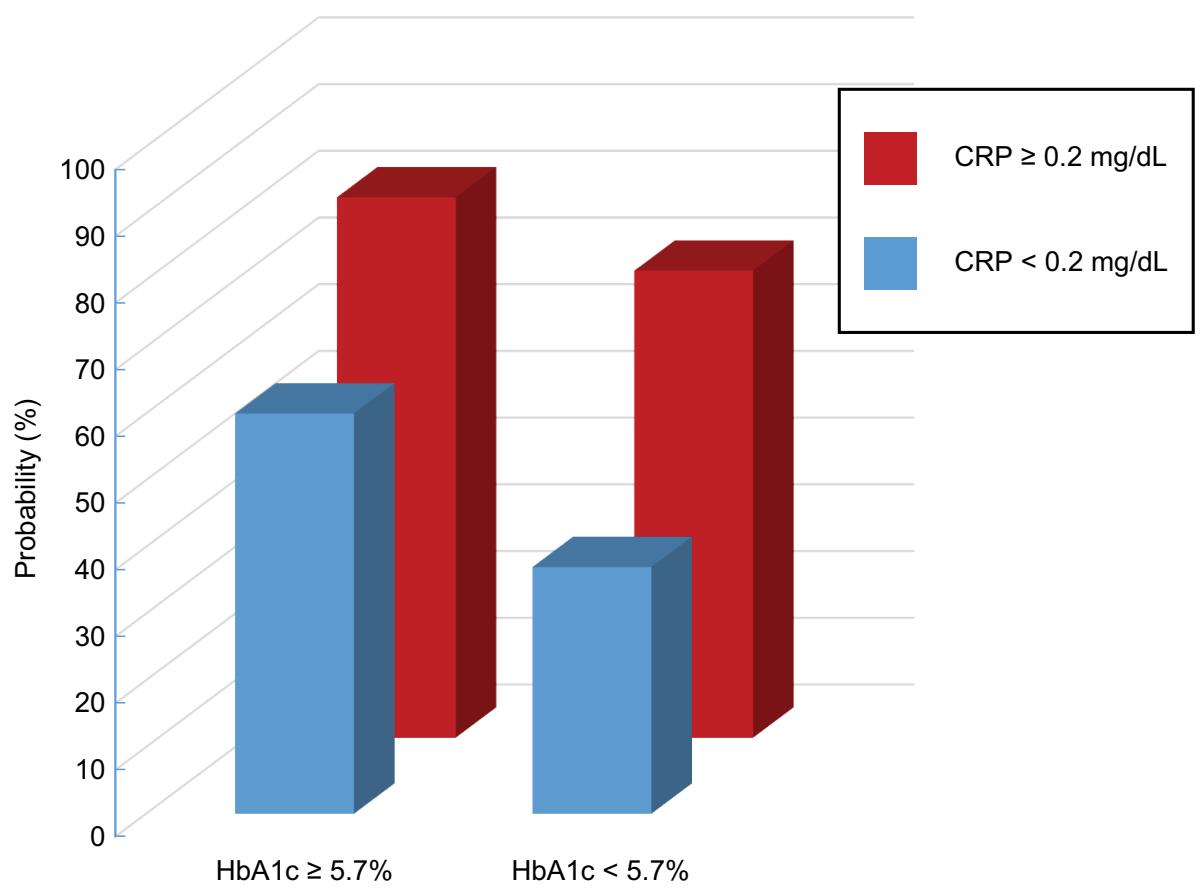

Figure I Probability of moderate/severe OSA by CRP and HbAIc values in combination.

Notes: When CRP was $\geq 0.2 \mathrm{mg} / \mathrm{dL}$ and $\mathrm{HbAlc} \geq 5.7 \%$, the probability of moderate/severe OSA was $81 \%$. When neither marker was elevated, the probability was $37 \%$, which was less than half that observed when both markers are elevated.

Abbreviations: CRP, C-reactive protein; OSA, obstructive sleep apnea.

\section{Probability of moderate/severe OSA using CRP and $\mathrm{HbAlc}$ in combination}

The probabilities of moderate/severe OSA by CRP and $\mathrm{HbAlc}$ values in combination are shown in Figure 1. The predictive value is additive when CRP and $\mathrm{HbA} 1 \mathrm{c}$ are combined. When $\mathrm{CRP} \geq 0.2 \mathrm{mg} / \mathrm{dL}$ and $\mathrm{HbA} 1 \mathrm{c} \geq 5.7 \%$, the probability was $81 \%$. When neither marker was elevated, the probability was $37 \%$. When only one of the two markers was elevated (but not both), the probability was $60 \%$ to $70 \%$.

\section{ROC curve analysis and AUC}

The ROC AUCs for the five individual biomarkers are shown in Table 2. The strongest predictors of OSA were CRP and $\mathrm{HbA1c}$.

\section{Biomarker combinations}

Algorithms generated potentially promising combinations of markers. A weighted linear combination (hereafter referred to as " $\mathrm{HbA} 1 \mathrm{c}+\mathrm{CRP}+\mathrm{EPO}$ ") with the formula $12.81 * \log (\mathrm{HbA} 1 \mathrm{c})$ 
$+0.75 * \log (\mathrm{CRP})+1.53 * \log (\mathrm{EPO})($ Note: CRP units are $\mathrm{mg} /$ $\mathrm{dL}$, and " $\log$ " refers to $\log$ base 10 ), yielded a 0.06 increase in AUC (0.78 [95\% CI 0.71-0.86]) over individual markers (HbA1c 0.68 [95\% CI 0.61-0.76]; CRP 0.72 [95\% CI $0.63-0.81$ ]; and EPO 0.67 [95\% CI 0.58-0.76]) for detection of OSA.

Table 2 Comparison of ROC AUCs for OSA: biomarkers and clinical measures

\begin{tabular}{llll}
\hline Category & Parameter & AUC & 95\% Cl \\
\hline Biomarkers & HbAIc+CRP+EPO & 0.78 & $0.7 \mathrm{I}-0.86$ \\
& HbAIc & 0.68 & $0.6 \mathrm{I}-0.76$ \\
& CRP & 0.72 & $0.63-0.8 \mathrm{I}$ \\
& EPO & 0.67 & $0.58-0.76$ \\
& IL-6 & 0.63 & $0.54-0.72$ \\
Clinical & Uric acid & 0.60 & $0.5 \mathrm{I}-0.70$ \\
measures & Age & 0.63 & $0.54-0.72$ \\
& BMI & 0.78 & $0.7 \mathrm{I}-0.85$ \\
& Neck circumference & 0.74 & $0.67-0.8 \mathrm{I}$ \\
& Systolic BP & 0.59 & $0.45-0.72$ \\
& Diastolic BP & 0.51 & $0.40-0.62$ \\
& STOP-Bang & 0.70 & $0.61-0.80$ \\
& Epworth Sleepiness Scale (ESS) & 0.53 & $0.42-0.64$ \\
\hline
\end{tabular}

Abbreviations: AUC, area under the curve; BMI, body mass index; BP, blood pressure; CRP, C-reactive protein; EPO, erythropoietin; OSA, obstructive sleep apnea; ROC, receiver operating characteristic.
This validates the results of our feasibility study which demonstrated that a combination of markers provides superior diagnostic utility to individual markers alone; the feasibility study found that the combination $\mathrm{HbAlc}+\mathrm{CRP}+\mathrm{EPO}$ yielded a 0.07 increase in AUC (0.82 [95\% CI 0.72-0.91]) over individual markers (HbA1c 0.74 [95\% CI 0.63-0.86]; CRP 0.67 [95\% CI 0.55-0.80]; and EPO 0.68 [95\% CI 0.55-0.80]) for detection of OSA. A similar pattern of results, with the biomarker combination yielding a higher AUC than the individual markers, was observed for detection of moderate/ severe OSA. ${ }^{27}$

Table 2 shows that the point estimate for the AUC for the combination of three markers in the current study (0.78) was higher than all individual biomarkers, STOP-Bang (0.70), and ESS (0.53) scores, and all clinical measures apart from BMI (0.78). However, in non-obese subjects $(\mathrm{BMI}<30, \mathrm{n}=109)$, the combination of biomarkers (0.75) was higher than BMI (0.61), as well as the STOP-Bang (0.66) and ESS (0.56) scores (Figure 2).

The combination of three markers performed comparably in asymptomatic (ESS $\leq 10, \mathrm{n}=144$; AUC 0.76) and symptomatic (ESS >10, n=120; AUC 0.73) patients.

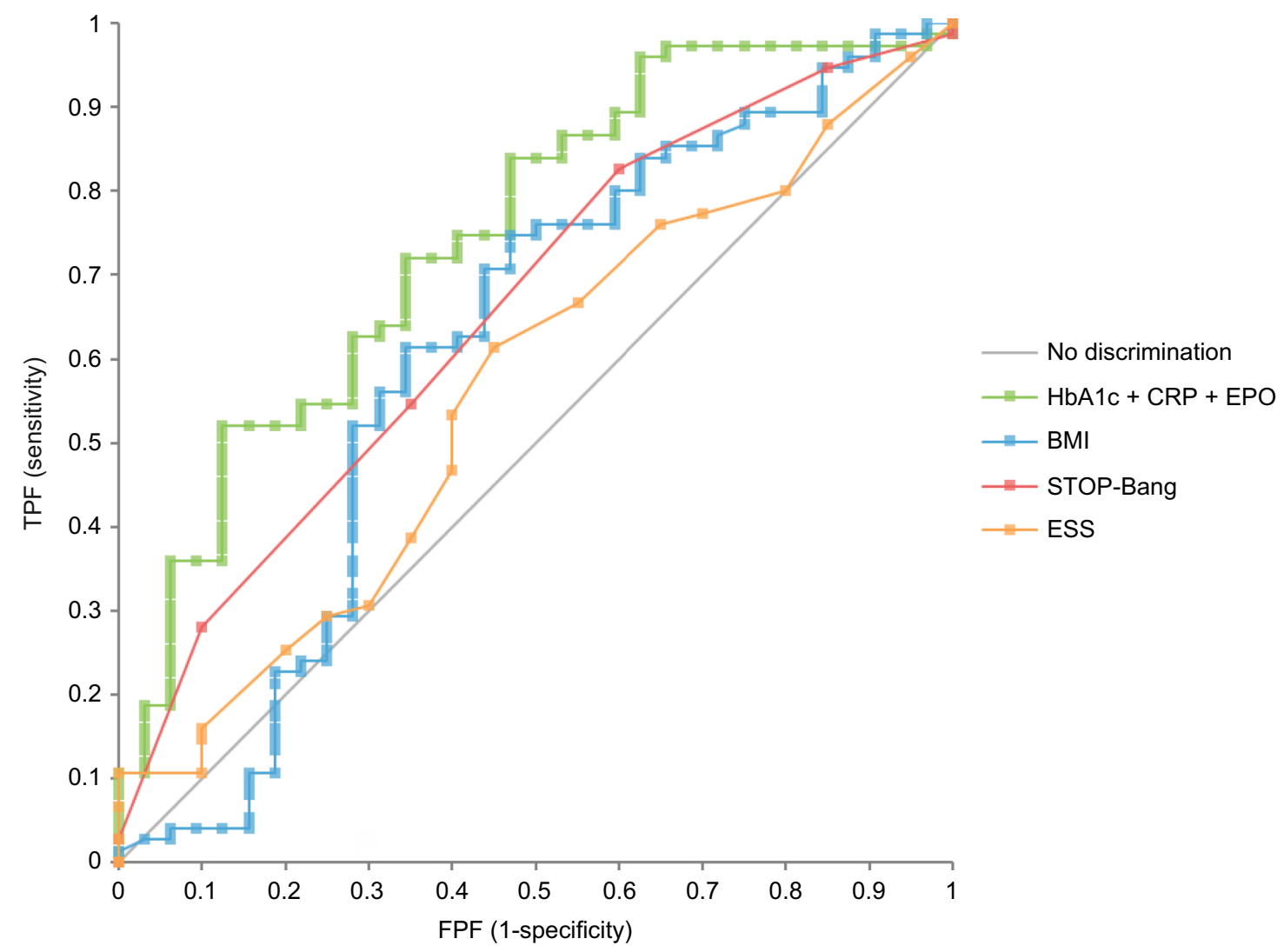

Figure 2 ROC curves for detection of OSA in non-obese subjects (BMI <30).

Notes: In non-obese subjects, the AUC for the combination of biomarkers (0.75) was higher than BMI (0.6I), as well as the STOP-Bang (0.66) and ESS (0.56) scores. Abbreviations: AUC, area under the curve; BMI, body mass index; BP, blood pressure; CRP, C-reactive protein; EPO, erythropoietin; ESS, Epworth Sleepiness Scale; FPF, false positive fraction; OSA, obstructive sleep apnea; ROC, receiver operating characteristic; TPF, true positive fraction. 


\section{Sensitivity, specificity, and cutoff for detection of OSA}

The combined biomarkers correctly identified a greater percentage of OSA patients (sensitivity) and non-OSA patients (specificity) compared to the ESS and STOPBang questionnaires. Defining the optimal cutoff as one that yields approximately $80 \%$ sensitivity, the following sensitivity and specificity results, respectively, were obtained for $\mathrm{HbA} 1 \mathrm{c}+\mathrm{CRP}+\mathrm{EPO}(81 \%$ and $60 \%)$, ESS $(78 \%$ and $19 \%)$, and STOP-Bang (75\% and 52\%). Furthermore, $\mathrm{HbA} 1 \mathrm{c}+\mathrm{CRP}+\mathrm{EPO}$ provided comparable sensitivity and higher specificity compared to BMI in all subjects $(81 \%$ and $56 \%$ ), and particularly in non-obese subjects $(81 \%$ and $38 \%$ ). The biomarker combination weighted formula $\mathrm{HbA1c}+\mathrm{CRP}+\mathrm{EPO}$ cutoff that yielded these results is 9.95 .

Defining optimal cutoffs as those commonly used in clinical practice, Table 3 shows measures of diagnostic test performance for key parameters.

\section{Correlation with severity of disease}

The combination of three biomarkers increased with increasing severity of disease. $\mathrm{HbAlc}+\mathrm{CRP}+\mathrm{EPO}$ median values (IQR) were as follows: Controls 9.47 (9.30-9.91), NonOSA 9.90 (9.49-10.35), Mild-OSA 10.28 (9.74-10.75), Moderate-OSA 10.50 (10.00-10.83), and Severe-OSA 10.74 (10.34-11.62) $(p<0.0001$ for all).

Correlation coefficients were calculated to evaluate the relationship between the $\mathrm{HbA} 1 \mathrm{c}+\mathrm{CRP}+\mathrm{EPO}$ biomarker combination and measures of OSA severity (AHI, minimum oxygen saturation, and percentage cumulative sleep time with oxygen saturation $<90 \%$ ). Significant correlations were observed $(r=0.40 p<0.0001 ; r=-0.40 p<0.0001 ; r=40$ $p<0.0001$ ) for all three measures of severity.

\section{Odds ratios}

Multivariate logistic regression models tested the association between possible risk factors and OSA. Unadjusted statistically significant predictors of OSA included age (OR 2.58, $p=0.035$ ), BMI (OR 4.31, $p=0.0005)$, and HbA1c+CRP+EPO (OR 5.18, $p<0.0001)$. In multiple logistic regression models adjusted for age and BMI, $\mathrm{HbA} 1 \mathrm{c}+\mathrm{CRP}+\mathrm{EPO}$ remained significantly associated with OSA (OR 3.36, $p=0.005$ ) whereas the adjusted OR for age was $1.97(p=0.16)$ and BMI was $3.41(p=0.005)$.

\section{Discussion}

The objective of this multicenter prospective study was to evaluate the use of blood biomarkers, individually and in combination, to screen for OSA in adult men, to test the hypothesis that clusters of biomarkers may provide a characteristic signature of OSA. This validation study was conducted in order to replicate findings from a prior feasibility study. ${ }^{27}$ When using biomarker panels and algorithms, reproducibility is essential to establishing the correct biomarkers and formulas. Two studies were required in two independent cohorts - a feasibility study and a validation study. The earlier feasibility study served to generate a hypothesis, and the current validation study is the pivotal trial demonstrating reproducibility of results and establishing the relevant combination of biomarkers and the algorithm formula.

We found that the most predictive individual OSA biomarkers were CRP and $\mathrm{HbAlc}$, replicating findings from the earlier feasibility study. ${ }^{27}$ The ROC curve analysis for HbAlc and CRP found that these individual biomarkers showed an advantage over existing OSA screening tools, particularly the ESS questionnaire (AUCs $0.68,0.72$, and 0.53 , respectively). Furthermore, we demonstrate that a combination of $\mathrm{HbA1}+\mathrm{CRP}+\mathrm{EPO}$ yielded a higher AUC $(0.78)$ than individual markers alone and was more predictive than ESS (0.53) and STOP-Bang (0.70) questionnaires. The diagnostic utility of the combination biomarker persisted after adjusting for age, BMI, and subjective sleepiness. Thus, our identified combination biomarker provides greater diagnostic utility than common OSA screening questionnaires, and may assist primary care providers in identifying at-risk patients requiring sleep study referral.

Table 3 Measures of diagnostic test performance for key parameters

\begin{tabular}{|c|c|c|c|c|c|c|c|}
\hline Parameter & Cutoff $(\geq)$ & Sensitivity & Specificity & PPV & NPV & LR(+) & LR(-) \\
\hline $\mathrm{HbAlc}+\mathrm{CRP}+\mathrm{EPO}$ & 9.95 & 81 & 60 & 91 & 39 & 2.06 & 0.31 \\
\hline $\mathrm{HbAlc}$ & $5.7 \%$ & 38 & 88 & 94 & 22 & 3.26 & 0.70 \\
\hline CRP & $0.2 \mathrm{mg} / \mathrm{dL}$ & 52 & 77 & 92 & 24 & 2.22 & 0.63 \\
\hline EPO & $8 \mathrm{mlU} / \mathrm{mL}$ & 66 & 58 & 89 & 25 & 1.58 & 0.58 \\
\hline BMI & 30 & 64 & 78 & 94 & 30 & 2.93 & 0.46 \\
\hline Neck Circumference & 17 in & 44 & 93 & 97 & 25 & 6.36 & 0.60 \\
\hline STOP-Bang & 3 & 91 & 29 & 87 & 39 & 1.29 & 0.30 \\
\hline STOP-Bang & 5 & 44 & 84 & 93 & 23 & 2.73 & 0.67 \\
\hline ESS & 11 & 53 & 55 & 86 & 19 & 1.18 & 0.85 \\
\hline
\end{tabular}

Abbreviations: BMI, body mass index; CRP, C-reactive protein; EPO, erythropoietin; ESS, Epworth Sleepiness Scale; PPV, positive predictive value; NPV, negative predictive value; LR, likelihood ratio. 
OSA is a highly prevalent medical condition that remains underdiagnosed. The AASM reports blood biomarkers may be useful in identifying individuals at risk for OSA. ${ }^{12}$ Implementation of OSA biomarker screening by primary care providers in high-risk populations could significantly improve the accuracy of sleep specialist referrals and ultimately improve patient outcomes. ${ }^{29}$ The biomarker combination correlates with OSA severity, with concomitant elevation of $\mathrm{HbA} 1 \mathrm{c}$ and CRP indicating a high probability of moderate/severe OSA ( $81 \%)$; this probability fell to less than half $(37 \%)$ when neither marker was elevated. Use of the $\mathrm{HbA} 1 \mathrm{c}+\mathrm{CRP}+\mathrm{EPO}$ biomarker combination (higher values indicate more serious disease), and use of probability estimates from $\mathrm{HbAlc}$ and $\mathrm{CRP}$, provides two methods to identify more serious moderate/severe disease. The AUC for the combination of markers $(0.78)$ was higher than all clinical measures except for BMI (0.78). However, in non-obese subjects $(\mathrm{BMI}<30)$, the combination of biomarkers $(0.75)$ was higher than BMI (0.61), STOP-Bang (0.66), and ESS (0.56) scores. Given that one-third to one-half of patients ultimately diagnosed with OSA are not obese, ${ }^{30,31}$ up to $90 \%$ of individuals with OSA remain undiagnosed, ${ }^{2-5}$ and $34 \%$ of men have this disorder; ${ }^{1}$ a successful screening test such as the combination biomarker that identifies non-obese men with OSA would represent a major opportunity to improve the diagnosis of millions of men with this highly prevalent condition. Future studies examining this non-obese population in detail would further validate our findings. Prior studies lend support to our results and have reported inflammatory biomarkers to be significantly higher in OSA patients independent of obesity. ${ }^{32}$

Our study is the first to comprehensively assess the diagnostic accuracy of a novel combination of blood biomarkers in identifying patients with suspected OSA. Although the markers with the highest predictive value (HbA1c, CRP, and EPO) in our study have previously been associated individually with OSA, they have not been evaluated as a signature cluster, and few prior studies report ROC analyses with sensitivity and specificity results. ${ }^{18}$

The high diagnostic performance of these specific markers is not unexpected. Prior studies have reported independent associations between OSA, insulin resistance, and diabetes, ${ }^{33}$ with mild to moderate elevations in HbA1c $(\geq 5.7 \%)$ suggestive of prediabetes. Prediabetes is associated with disorders typically only considered with diabetes, such as cardiovascular disease, hypertension, and metabolic syndrome. ${ }^{34}$ Elevated CRP levels $(\geq 0.2 \mathrm{mg} / \mathrm{dL})$ are associated with intermediate to high risk of heart disease, with reported independent associations between OSA and cardiovascular disease. ${ }^{6}$
OSA-induced hypoxemia is associated with increased EPO concentrations, ${ }^{25}$ and EPO decreases after continuous positive airway pressure (CPAP) therapy in those with OSA. ${ }^{25,35}$ Healthy individuals subjected to hypoxemia show increased EPO ${ }^{36}$ EPO promotes the formation of red blood cells; the mild EPO elevations ( $\geq 8 \mathrm{mIU} / \mathrm{mL}$ ) observed in OSA patients in this study may represent a response to hypoxemia. Therefore, there is a biological plausibility for including EPO in the biomarker panel for OSA.

\section{Study limitations}

Our study results provide a new potential methodology to improve the accuracy of screening for OSA, and suggest areas for further research. Studies in large cohorts, particularly population-based studies, are required to further validate our study findings, and to evaluate the diagnostic utility in additional patient subgroups, including women. These larger studies could also evaluate biomarker algorithms incorporating additional clinical data such as BMI, snoring, and sleepiness. In addition, monitoring studies may be useful to assess the utility of the individual biomarkers and a combination of biomarkers in measuring compliance and response to therapy. Health economic studies assessing the cost-effectiveness of biomarker screening in broader populations, and whether the biomarker results motivate patients to follow up with a sleep study referral, may also be useful.

\section{Conclusion}

OSA-associated blood biomarkers in combination provide a new approach to screening for OSA. The biomarker combination $\mathrm{HbA} 1 \mathrm{c}+\mathrm{CRP}+\mathrm{EPO}$ was diagnostically superior to ESS and tended to be more predictive than the STOP-Bang questionnaire. In non-obese subjects (up to $50 \%$ of OSA patients are non-obese), the biomarker combination was superior to BMI in predicting OSA. The combination performed well in both asymptomatic and symptomatic patients. The results of this study verify our hypothesis and replicate findings from a prior feasibility study in an independent cohort that show OSA is associated with a characteristic signature cluster of biomarker changes. As mentioned in Study Limitations, population-based studies would assist with validation of the algorithm before use in general medical practice. Concurrent elevations of HbA1c, CRP, and EPO levels should generate a high index of OSA suspicion and, thus, may be useful as an initial OSA screening tool, which could subsequently lead to a sleep study referral. Combinations of biomarkers correlate with severity of disease and, therefore, may also assist sleep centers in identifying and triaging higher risk patients for sleep study diagnosis and treatment. 


\section{Acknowledgments}

The authors are grateful to the sleep center staff for their expertise and technical assistance in data collection and participation in the study. Beckman Coulter has a patent pending: Obstructive Sleep Apnea (OSA) Biomarker Panel.

\section{Disclosure}

This work was supported by research grant funding from Beckman Coulter, Inc. (Brea, CA, USA) provided to the respective institutions of Drs. Fleming, Holty, Bogan, Hwang, Ferouz-Colborn, Budhiraja, Redline, Mensah-Osman, Osman, and Li. Drs. Samoszuk, Riley, and Southwick, as well as Ms. Cruz, Mr. Bai, and Mr. Lu are employed by Beckman Coulter. Dr. Azad and Ms. Podolak report no conflicts of interest in this work.

\section{References}

1. Peppard PE, Young T, Barnet JH, Palta M, Hagen EW, Hla KM. Increased prevalence of sleep-disordered breathing in adults. Am J Epidemiol. 2013;177(9):1006-1014.

2. Watson NF. Health care savings: the economic value of diagnostic and therapeutic care for obstructive sleep apnea. J Clin Sleep Med. 2016;12(8):1075-1077.

3. Bousquet J, Khaltaev N, eds. World Health Organization. Global surveillance, prevention and control of chronic respiratory diseases: a comprehensive approach. Geneva: WHO; 2007. Available from: http:/www.who.int/gard/publications/GARD\%20Book\%202007.pdf. Accessed February 03, 2018.

4. Kapur V, Strohl KP, Redline S, Iber C, O’Connor G, Nieto J. Underdiagnosis of sleep apnea syndrome in U.S. communities. Sleep Breath. 2002;6(2):49-54.

5. Young T, Evans L, Finn L, Palta M. Estimation of the clinically diagnosed proportion of sleep apnea syndrome in middle-aged men and women. Sleep. 1997;20(9):705-706.

6. St-Onge MP, Grandner MA, Brown D, et al; American Heart Association Behavior Change, Diabetes, and Nutrition Committees of the Council on Lifestyle and Cardiometabolic Health; Council on Cardiovascular Disease in the Young; Council on Clinical Cardiology; and Stroke Council. Sleep duration and quality: impact on lifestyle behaviors and cardiometabolic health: a scientific statement from the American Heart Association. Circulation. 2016;134:367-386.

7. Redline S. Screening for obstructive sleep apnea: implications for the sleep health of the population. JAMA. 2017;317(4):368-370.

8. Budhiraja R, Budhiraja P, Quan SF. Sleep-disordered breathing and cardiovascular disorders. Respir Care. 2010;55(10):1322-1332.

9. Punjabi NM, Caffo BS, Goodwin JL, et al. Sleep-disordered breathing and mortality: a prospective cohort study. PLoS Med. 2009;6(8):e1000132.

10. Aurora RN, Punjabi NM. Obstructive sleep apnoea and type 2 diabetes mellitus: a bidirectional association. Lancet Respir Med. 2013;1(4):329-338.

11. Redline S, Yenokyan G, Gottlieb DJ, et al. Obstructive sleep apneahypopnea and incident stroke: the sleep heart health study. Am J Respir Crit Care Med. 2010;182(2):269-277.

12. Kapur VK, Auckley DH, Chowdhuri S, et al. Clinical practice guideline for diagnostic testing for adult obstructive sleep apnea: an American Academy of Sleep Medicine clinical practice guideline. J Clin Sleep Med. 2017;13(3):479-504.

13. Kapur V, Blough DK, Sandblom RE, et al. The medical cost of undiagnosed sleep apnea. Sleep. 1999;22(6):749-755.

14. Kakkar RK, Berry RB. Positive airway pressure treatment for obstructive sleep apnea. Chest. 2007;132(3):1057-1072.
15. Kapur VK. Obstructive sleep apnea: diagnosis, epidemiology, and economics. Respir Care. 2010;55(9):1155-1167.

16. Surani SR. Diabetes, sleep apnea, obesity and cardiovascular disease: Why not address them together? World J Diabetes. 2014;5(3):381-384.

17. Qaseem A, Dallas P, Owens DK, Starkey M, Holty JE, Shekelle P; Clinical Guidelines Committee of the American College of Physicians. Diagnosis of obstructive sleep apnea in adults: a clinical practice guideline from the American college of physicians. Ann Intern Med. 2014;161(3):210-220.

18. De Luca Canto G, Pachêco-Pereira C, Aydinoz S, Major PW, FloresMir C, Gozal D. Biomarkers associated with obstructive sleep apnea: a scoping review. Sleep Med Rev. 2015;23:28-45.

19. Maeder MT, Mueller C, Schoch OD, Ammann P, Rickli H. Biomarkers of cardiovascular stress in obstructive sleep apnea. Clin Chim Acta. 2016;460:152-163.

20. Montesi SB, Bajwa EK, Malhotra A. Biomarkers of sleep apnea. Chest. 2012;142(1):239-245.

21. De Luca Canto G, Pachêco-Pereira C, Aydinoz S, Major PW, Flores-Mir C, Gozal D. Diagnostic capability of biological markers in assessment of obstructive sleep apnea: a systematic review and meta-analysis. J Clin Sleep Med. 2015;11(1):27-36.

22. Papanas N, Steiropoulos P, Nena E, Tzouvelekis A, Maltezos E, Trakada $\mathrm{G}$, Bouros D. HbA1c is associated with severity of obstructive sleep apnea hypopnea syndrome in nondiabetic men. Vasc Health Risk Manag. 2009;5:751-756.

23. Punjabi NM, Beamer BA. C-reactive protein is associated with sleep disordered breathing independent of adiposity. Sleep. 2007;30(1): 29-34.

24. Yokoe T, Minoguchi K, Matsuo H, et al. Elevated levels of C-reactive protein and interleukin-6 in patients with obstructive sleep apnea syndrome are decreased by nasal continuous positive airway pressure. Circulation. 2003;107(8):1129-1134.

25. Winnicki M, Shamsuzzaman A, Lanfranchi P, et al. Erythropoietin and obstructive sleep apnea. Am J Hypertens. 2004;17(9):783-786.

26. Hirotsu C, Tufik S, Guindalini C, Mazzotti DR, Bittencourt LR, Andersen ML. Association between uric acid levels and obstructive sleep apnea syndrome in a large epidemiological sample. PLoS One. 2013;8(6):e66891.

27. Fleming WE, Ferouz-Colborn A, Samoszuk MK, et al. Blood biomarkers of endocrine, immune, inflammatory, and metabolic systems in obstructive sleep apnea. Clin Biochem. 2016;49(12):854-861.

28. Berry RB, Brooks R, Gamaldo CE, et al; American Academy of Sleep Medicine. The AASM Manual for the Scoring of Sleep and Associated Events: Rules, Terminology and Technical Specifications. Version 2.3. Darien, IL: American Academy of Sleep Medicine; 2016.

29. Aurora RN, Quan SF. Quality measure for screening for adult obstructive sleep apnea by primary care physicians. J Clin Sleep Med. 2016;12(8): 1185-1187.

30. Punjabi NM, Sorkin JD, Katzel LI, Goldberg AP, Schwartz AR, Smith PL. Sleep-disordered breathing and insulin resistance in middle-aged and overweight men. Am J Respir Crit Care Med. 2002;165(5):677-682.

31. Vgontzas AN, Papanicolaou DA, Bixler EO, et al. Sleep apnea and daytime sleepiness and fatigue: relation to visceral obesity, insulin resistance and hypercytokinemia. J Clin Endocrinol Metab. 2000;85(3): 1151-1158.

32. Thunström E, Glantz H, Fu M, et al. Increased inflammatory activity in nonobese patients with coronary artery disease and obstructive sleep apnea. Sleep. 2015;38(3):463-471.

33. Botros N, Concato J, Mohsenin V, Selim B, Doctor K, Yaggi HK. Obstructive sleep apnea as a risk factor for type 2 diabetes. Am J Med. 2009;122(12):1122-1127.

34. Buysschaert M, Medina JL, Bergman M, Shah A, Lonier J. Prediabetes and associated disorders. Endocrine. 2015;48(2):371-393.

35. Cahan C, Decker MJ, Arnold JL, Goldwasser E, Strohl KP. Erythropoietin levels with treatment of obstructive sleep apnea. J Appl Physiol (1985). 1995;79(4):1278-1285.

36. Knaupp W, Khilnani S, Sherwood J, Scharf S, Steinberg H. Erythropoietin response to acute normobaric hypoxia in humans. J Appl Physiol (1985). 1992;73(3):837-840. 


\section{Supplementary material}

Table SI Full title of each ethical review board, and its affiliated institution

\begin{tabular}{|c|c|}
\hline Institution & Institutional Review Board \\
\hline Sleep Center Orange County, Irvine, CA, USA & Western Institutional Review Board, Puyallup, WA, USA \\
\hline Stanford Medical School, VA Palo Alto Health Care System & Administrative Panel on Human Subjects in Clinical Research, Stanford \\
\hline $\begin{array}{l}\text { Pulmonary, Critical Care and Sleep Medicine Section, Palo Alto, CA, } \\
\text { USA }\end{array}$ & Institutional Review Board, Palo Alto, CA, USA \\
\hline SleepMed Inc., Bogan Sleep Consultants, LLC, Columbia, SC, USA & Western Institutional Review Board, Puyallup, WA, USA \\
\hline $\begin{array}{l}\text { Southern California Permanente Medical Group, Sleep Medicine } \\
\text { Department, Kaiser Permanente, Fontana Medical Center, Fontana, CA, } \\
\text { USA }\end{array}$ & $\begin{array}{l}\text { Kaiser Permanente Southern California Institutional Review Board, } \\
\text { Pasadena, CA, USA }\end{array}$ \\
\hline $\begin{array}{l}\text { Brigham and Women's Hospital, Harvard Medical School, Division of } \\
\text { Sleep Medicine, Boston, MA, USA }\end{array}$ & Partners Human Research Committee, Somerville, MA, USA \\
\hline $\begin{array}{l}\text { EENA Comprehensive Neurology \& Sleep Center, Boynton Beach, FL, } \\
\text { USA }\end{array}$ & EENA Institutional Review Board, Boynton Beach, FL, USA \\
\hline $\begin{array}{l}\text { South Bend Medical Foundation (Laboratory Testing), New Technology } \\
\text { and Test Development, South Bend, IN, USA }\end{array}$ & Schulman Institutional Review Board, Cincinnati, OH, USA \\
\hline Medical Research Networx, LLC. (CRO), West Wareham, MA, USA & Diagnostics Institutional Review Board, Cummaquid, MA, USA \\
\hline
\end{tabular}

\section{Publish your work in this journal}

Nature and Science of Sleep is an international, peer-reviewed, open access journal covering all aspects of sleep science and sleep medicine, including the neurophysiology and functions of sleep, the genetics of sleep, sleep and society, biological rhythms, dreaming, sleep disorders and therapy, and strategies to optimize healthy sleep. The manuscript

\section{Dovepress}

management system is completely online and includes a very quick and fair peer-review system, which is all easy to use. Visit http://www. dovepress.com/testimonials.php to read real quotes from published authors. 